\title{
A Short Note on Alternative Strong Force
}

\author{
Mukul Chandra Das ${ }^{1 *}$, Rampada Misra ${ }^{2}$ \\ ${ }^{1}$ Singhania University, Rajasthan, India \\ ${ }^{2}$ Department of Electronics, Vidyasagar University, Paschim Midnapur, India \\ Email: *mukuldas.100@gmail.com
}

Received March 18, 2012; revised March 31, 2012; accepted April 20, 2012

\begin{abstract}
All nucleons are concentrated in an infinitesimal region in the atom under the strong force forming the collective model. Mathematically, formation of this force has been explained by H. Yukawa in [1]. But, in this text, this force has been derived following an alternative and constructive method (or system) which also leads to explain the generalized nuclear model.
\end{abstract}

Keywords: Strong Gravitational Force; Superimposed Spins

\section{Introduction}

It is well known to us that spin is the intrinsic property of elementary particles. Again, energy, mass, moment of inertia etc of a particle of rigid configuration would be affected by relativistic spin as in [2]. In [3] it is clear that a particle or system can possess three simultaneous superimposed rotational motional as well as three simultaneous superimposed spins to the view of an observer. Again in [4] considering S-S or S-L or L-L interaction, relation between electromagnetic field and gravitational field has been explained as $\psi_{\alpha}=\Upsilon w_{i j} G_{\alpha}^{\prime}$.

In the present work trial would be made to derive the formation of strong force in the nucleus by extending the above relation.

\section{Strong Force}

According to [4] it is known to us that

$$
\psi_{\alpha}=\Upsilon w_{i j} G_{\alpha}^{\prime}
$$

From the above equation we can write

$$
G_{\alpha}^{\prime}=\Upsilon^{-1} w_{i j}^{-1} \psi_{\alpha}
$$

Again from (1) and (2) using a constant (K) we can consider a relation as

$$
G_{\alpha}=\mathrm{K} w_{i j} \psi_{\alpha}^{\prime}
$$

This means that electromagnetic field performing two simultaneous superimposed motions generate one kind of strong gravitational field which is related to and stronger than electromagnetic field of primed frame. Now let,

$$
\psi_{\alpha}^{\prime}=\Upsilon w_{i j}^{\prime} G_{\alpha}^{\prime \prime}
$$

"Corresponding author.
Then using (3) and (4) we get

$$
G_{\alpha}=\Gamma w_{i j} w_{i j}^{\prime} G^{\prime \prime}
$$

This is the relation between strong field and well known gravitational field. Here, $G^{\prime \prime}$ performing two simultaneous superimposed motions as in [4] generates $\psi_{\alpha}^{\prime}$. Again $\psi_{\alpha}^{\prime}$ performing this type of motions generates $G_{\alpha}$ to an observer. Now, energy of strong gravitational field is the kinetic energy due to relativistic movement of electromagnetic mass as well as protons. So,

$$
E_{G}=E_{e m}(\gamma-1)
$$

where, $E_{G}$ is the strong gravitational energy and $E_{e m}$ electromagnetic energy.

$$
\gamma=1 / \sqrt{1-w^{2} / c^{2}}
$$

$w$ being the four velocity as in [4]. It is well known that nucleus possesses spin. But, it is more significant that the nucleus should possess two simultaneous superimposed spins. Therefore, electromagnetic field of protons performing two simultaneous superimposed motions generates such a field which is strong enough to concentrate the nucleons against their repulsive force. It is also pointed out that due to such superimposed spins of nucleus, different shells $(n=1,2,3 \cdots)$ and sub-shells $(s, p, d \cdots)$ also are formed by the nucleons. On the basis of $n, l, J$ (three quantum number) as in [1] energy levels in the nucleus are

$$
1 s_{1 / 2}, 1 p_{3 / 2}, 1 p_{1 / 2}, 1 d_{5 / 2}, 1 d_{3 / 2}, 2 s_{1 / 2} \cdots 2 f_{7 / 2}, 2 f_{5 / 2} \text { etc. }
$$

Number of maximum nucleons in a particular $J$ sub energy level is $(2 J+1)$.

Using (6) we get energy of strong field which is same 
as binding energy $\left(E_{B}\right)$ in nucleus. So,

$$
E_{B}=\sum_{n=1,2,3 \cdots} E_{B}^{n}
$$

and

$$
E_{B}^{n}=e_{e m}\left[A_{s}^{n}\left(\gamma_{s}^{n}-1\right)+A_{p}^{n}\left(\gamma_{p}^{n}-1\right)+\cdots\right]
$$

where, $A_{s}^{n}, A_{p}^{n} \cdots$ are number of protons in the respective sub-shells of $n$th shell and $e_{e m}$ is the electromagnetic energy attributed to the proton.

\section{Conclusion}

It is seen that using four velocity matrix $w_{i j}$ we can transform electromagnetic field to strong field as in (3); also from weak gravitational field to strong field as in (5). Nucleus achieves collective model but, to explain the strong field, it will be more significant that nucleus possesses two simultaneous superimposed spins which forms the shell model. Thus, we may conclude that both shell model and collective model exist for a nucleus which is called generalized model.

\section{Acknowledgements}

Author thanks the authorities of Satmile High School, Satmile-721452, W. B., India for their continuous encouragements.

\section{REFERENCES}

[1] S. N. Ghoshal, "Nuclear Physics,” S. Chand \& Company Limited, New Delhi, 2006, pp. 365-430, 881-889.

[2] M. C. Das and R. Misra, "Relativistic Spin of an Arbitrary Body,” Advance Studies in Theoretical Physics, Vol. 6, No. 3, 2012, pp. 135-138.

[3] M. C. Das and R. Misra, "Three Lorentz Transformations Considering Two Rotations,” Advance Studies in Theoretical Physics, Vol. 6, No. 3, 2012, pp. 139-145.

[4] M. C. Das and R. Misra, "Fundamental Way of Charge Formation and Relation between Electromagnetic Field and Gravitational Field,” International Journal of Astronomy and Astrophysics, Vol. 2, No. 2, 2012. 